\title{
Weighting Iterative Fourier Transform Algorithm for Kinoform Implemented with Liquid-Crystal SLM
}

\author{
Alexander Kuzmenko', Pavlo Iezhov ${ }^{2}$ and Jin-Tae Kim ${ }^{3}$ \\ ${ }^{1}$ Institute of Applied Optics, 04053, Kyiv \\ 2Institute of Physics, 680028, Kyiv \\ ${ }^{3}$ Chosun University, 501-759, Gwangju \\ 1,2Ukraine \\ ${ }^{3}$ South Korea
}

\section{Introduction}

One of the most important trends in digital holography is the synthesis (calculation and fabrication) of diffraction optical elements (DOEs) serving for the transformation of a given light distribution into another light distribution with the desired characteristics (Bryngdahl \& Wyrowski, 1990). In the case where both the amplitude and the phase of output emission are of importance, the DOE is a digital hologram which can be binary amplitude, phase, or amplitude-phase (Lohmann \& Paris, 1967; Lee, 1979; Wyrowski \& Bryngdahl, 1988; Wyrowski, 1990-1991). But if we are interested only in the output emission intensity, then DOEs are synthesized as a purely phase structure of the kinoform type (Lesem et al., 1967; Hirsch et al., 1971; Gallagher \& Liu, 1973; Akahori, 1986; Aagebal \& Wyrowski, 1997; Skeren et al., 2002).

As distinct from an ordinary optical or digital hologram, a kinoform has a rather high diffraction efficiency attaining at least 90 per cent for a continuous kinoform. Therefore, it attracts a significant attention of experts in the applied and calculation-theoretic aspects. Among a lot of uses of the kinoform, there are particularly three interesting applications such as beam splitting (fan-out), beam shaping, and pattern or image generation. Optical fan-out elements split a single laser beam into a one- or two-dimensional array of beams and are key components in many applications of modern optics such as parallel optical processing, free-space communication in optical computing (Herzig et al., 1990; Gale et al., 1992, 1993; Ehbets et al., 1992; Prongue et al., 1992; Mait \& Brenner, 1988), and fiber optic communication (Wyrowski \& Zuidema, 1994). Fan-out elements with a smooth periodic phase structure have a theoretical limit by diffraction efficiency which is close to $100 \%$ (Herzig et al., 1990). Beam shaping is most commonly used in high-energy laser applications to the processing of various materials and in the laser branding or photolithographic illumination. These applications often require minimal energy losses, implying the use of phase-only elements such as a kinoform (Dixit et al., 1994; Leger et al., 1994; Chen et al., 1994; Duparre et al., 1995; Xin Tan et al., 1995; Johansson \& Bengtsson, 2000; Liu \& Taghizaden, 2002). Playing the role of a generator of images, a kinoform serves for the 
reproduction, in the form of a light intensity distribution, of a real binary or half-tone function stored in a computer in the discrete form.

The calculation of the phase structure of a kinoform is a partial case of the solution of a phase problem in the so-called "two-intensity" statement which is formulated for a Fourier-kinoform as follows. Let the input data such as the real function of an object $f_{o}(x, y)$ and the modulus of some spectrum equal to $1(u, v)$ be given. It is necessary to determine such phase distributions $\varphi(x, y)$ and $\psi(u, v)$ which together with the input data form a Fourier-pair

$$
f_{0}(x, y) \exp [i \varphi(x, y)] \Leftarrow \mathfrak{J}^{ \pm 1} \Rightarrow \exp [i \psi(u, v)],
$$

where $\mathfrak{I}^{+1}, \mathfrak{I}^{-1}$ are the direct and inverse Fourier transformation, respectively. The obtained solutions $\varphi$ and $\psi$ describe, respectively, the object-oriented phase scatterer (diffuser) and a spectral distribution of phases which is registered then on the phase medium in the form of a kinoform. We note that the solution of the phase problem for a kinoform has a specific feature. In the classical two-intensity statement of the phase problem, a solution exists always, because the true amplitude of the spectrum of an object function $f_{0}(x, y)$ is used in the Fourier-plane (though the determination of a solution can be not an easy task). For a kinoform, we require that the spectrum amplitude be equal to $1(u, v)$ in all the cases irrespective of the form of a function $f_{o}(x, y)$. In other words, we set the spectrum amplitude. Therefore, strictly speaking, the frequency-bounded phase structure $\psi(u, v)$, whose Fourier-transformation will form the given distribution of intensities $\left.f_{0}(x, y)\right|^{2}$, should not obligatorily exist. Nevertheless, an approximate (and sufficiently exact) solution of the kinoform problem exists practically always, which is supported by the practice of calculations.

Many algorithms of solution of phase problems are available. Prior to the beginning of the 1970s, the solution of inverse problems (which include the phase problem as well) was mainly a prerogative of professional mathematicians, because it requires to use a complicated mathematical apparatus and to construct high-complexity calculation's algorithms (Tikhonov \& Arsenin, 1977; Inverse Source Problems in Optics, 1978). The situation was changed, when the mathematically simple and physically transparent projective iterative Fourier-transform (IFT) algorithms were developed in the 1970-1980s (Lesem et al., 1967; Gerchberg \& Saxton, 1972; Gallagher \& Liu, 1973; Fienup, 1980, 1982), and after the clarification of the mathematical nature of these algorithms (Youla \& Webb, 1982; Levi \& Stark, 1987; Catino et al., 1997). The family of IFT-algorithms follows the philosophy of the Gerchberg-Saxton algorithm known as the error-reduction (ER) algorithm. All of these algorithms incorporate a similar idea - to iterate between the spatial and frequency domains, while successively satisfying a set of constraints in both. We start with an arbitrary phase-only filter in the object domain multiplying the input object (the original image). After the Fourier transformation, we obtain a Fourier domain image and set the required Fourier intensity (actually, the magnitude), leaving the phase, as it is. The inverse Fourier transformation brings us back to the object domain. Since we demand a phase-only filter, we impose the intensity of the input object in this plane. Then we calculate the Fourier transform and return to the Fourier domain, and so on. Earlier and now, this simple efficient idea of ER-iterations gives a conceptual basis for the development of most iterative methods of solution of phase problems in such fields as coherent optics, optical astronomy, electron 
and X-ray microscopy, biophysics, etc., where one deals with the diffraction of a coherent emission.

In Section 2 we describe algorithms we have tested. Section 3 presents the results of computer simulations, and Section 4 gives the results of optical experiments. The final section is devoted to the conclusion of this chapter.

\section{Algorithms}

\subsection{Weighting IFT-algorithm}

It is known that the phase calculation for a kinoform with the help of the IFT-algorithm in its classical ER-version described above gives no satisfactory results. The algorithm's convergence stagnates rapidly, and the mean square error in a reconstructed image remains large. In the present chapter, we will discuss a modified IFT-algorithm of synthesis of a kinoform (Kuzmenko, 2006, 2008), whose principal single distinction from the classical ERalgorithm consists in the use of a new nonlinear operation of the processing of the field amplitude in the object plane. To clarify its application, the work of the algorithm is illustrated in Fig. 1.

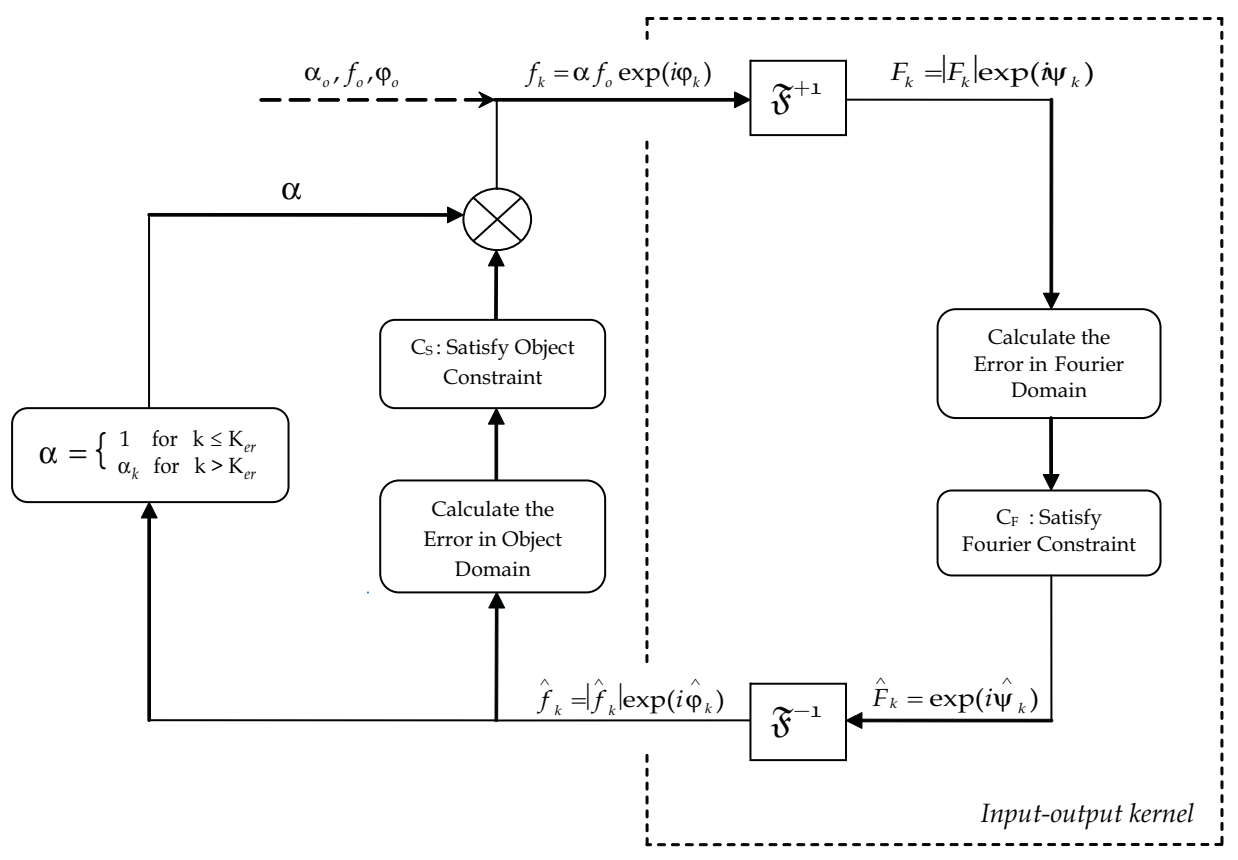

Fig. 1. Weighting IFT algorithm

First, one or several iterations $\left(K_{e r}\right)$ are realized by the classical ER scheme, in which the function $f_{o}$ is the ideal object, and $\varphi_{o}$ is an input phase scatterer imposed on it. Then, in all iterations with $k>K_{e r}$ at the formation of an input, the amplitude $f_{o}$ will be replaced by a new amplitude defined as 


$$
f_{k}=\alpha_{k} f_{o}
$$

where the weight coefficients $\alpha_{k}$ are determined by the recurrence relation

$$
\alpha_{k}=\alpha_{k-1} \beta_{k-1^{\prime}} \quad(k>1)
$$

where

$$
\beta_{k-1}=f_{o} /\left[\left|f_{k-1}^{\wedge}\right|+\varepsilon\right] .
$$

Here, $\left|\hat{f}_{k-1}\right|$ is the reconstructed amplitude on the $(k-1)-t h$ iteration, and $\varepsilon$ is a small number $\sim 10^{-10}$, excluding the division by zero. It should be mentioned that $f_{o}$ is real. The processing of the phases $\varphi_{k}$ and $\psi_{k}$ remains the same as that in the classical ER-algorithm. The phase of a kinoform $\psi_{k}$ can be quantized on each iteration with a required number of quantization levels in that case where it is necessary to study the quantization effects in the reconstructed image or to register a kinoform on a recording medium with a finite number of gradations of the phase.

Operations (2) - (4) are heuristic and have no strict mathematical justification. Their efficiency is established by extensive model and optical experiments. The physical sense of the coefficients $\alpha$ becomes clear if we consider the block of the algorithm separated by a dashed line in Fig ${ }_{\wedge} 1$, according to Fienup (Fienup, 1980, 1982), as a nonlinear unit with the input $f_{k}$, output $f_{k}$, and action operator $\mathfrak{I}^{+1} C_{F} \mathfrak{I}^{-1}$. Then, from the viewpoint of the theory of systems, the coefficients $\alpha$ is nothing but the matrix of coefficients of a negative feedback "output-input": if the amplitude $\left|f_{k-1}\right|$ on the $(k-1)$ - th iteration at some point $(x, y)$ of the plane of images is more than a given value $f_{o}$, then, on the next $k$-th iteration, the input $f_{o}$ at the corresponding point will be corrected. Namely, it will be decreased by $\alpha(x, y)$ times, and vice versa. At the same time, from the viewpoint of optics, the system of coefficients $\alpha$ normalized to one can be interpreted as some object-dependent amplitude filter which acts on the initial object $f_{0}$ and varies in the process of iterations. It is clear that, for all ER-iterations $\alpha(x, y)=\alpha_{0}(x, y)=1(x, y)$.

\subsection{Input-output algorithm}

In the course of experiments, we compared the weighting IFT-algorithm with the kinoform version of the Fienup input-output (IO) algorithm (Fienup, 1980). It is well-known and is one of the best at its estimation from the viewpoint of simplicity of the algorithm and the quality of a reconstructed image. Like the weighting algorithm, it differs from the ERalgorithm only by the mean of the processing of a field in the object plane. In the IO algorithm, after several preliminary ER-iterations, the input for the input-output kernel (see Fig. 1) for all subsequent iterations is taken in the form

$$
f_{k+1}=f_{k}+\mu\left\{2\left|f_{o}\right| \exp \left[i \hat{\varphi_{k}}\right]-\hat{f_{k}}-\left|f_{o}\right| \exp \left[i \varphi_{k}\right]\right\}
$$


or

$$
f_{k+1}=\hat{f_{k}}+\mu\left\{2\left|f_{o}\right| \exp \left[i \hat{\varphi_{k}}\right]-\hat{f_{k}}-\left|f_{o}\right| \exp \left[i \varphi_{k}\right]\right\}
$$

where $\mu$ is a free parameter, whose optimum value is selected experimentally. It is close to unity for half-tone objects and is usually in the interval 1.5-3.5 for binary objects. It should be noted that, in Eqs. (5) and (6), the previous input $f_{k}$ and the previous output $f_{k}$ serve, respectively, as a reference for the next input $f_{k+1}$. The term in the braces in both relations is a correction which must turn to zero, if the algorithm converges. Later on (Fienup, 1982), these two versions of the algorithm were named the input-output (IO) and output-output (OO) algorithms.

\section{Computer simulation}

A number of model experiments with various objects was realized with the purpose to study the potentialities of the weighting IFT-algorithm. Analogous experiments were performed also with the use of the IO and $\mathrm{OO}$ algorithms. In all the cases, the same phase starting diffuser $\varphi_{0}$ with a uniform distribution of phases in the interval $(0-2 \pi)$ is used. The variance of the amplitudes of images reconstructed in the process of iterations was evaluated as

$$
\sigma_{f}(k)=\frac{\sum_{l, m}\left[\left(f_{o}\right)_{l, m}-\chi(k)\left|\hat{f}_{l, m}(k)\right|\right]^{2}}{\sum_{l, m}\left(f_{o}\right)_{l, m}^{2}},
$$

where

$$
\chi(k)=\frac{\sum_{i, j}\left(f_{o}\right)_{i, j}^{2}}{\sum_{i, j}\left|\hat{f}_{i, j}(k)\right|^{2}}
$$

is the scale factor, the indices $l, m$ and $i, j$ run over the points, where the amplitude of an initial object $f_{o}$ is nonzero, and $k$ is the iteration number.

In the experiments involving the IO and $\mathrm{OO}$ algorithms, we used the optimum value of the object-depended coefficient $\mu_{o p t}$ in the equations (5) and (6), which provides the best convergence. The value of $\mu_{\text {opt }}$ was determined by means of the cyclic repetition of the procedure of synthesis for various values of $\mu$ (from the interval 0.1-5.0 with a step of 0.1). In Figs. 2 to 6, the results of model experiments on the synthesis of the kinoforms of binary and half-tone objects with a dimension of $64 \times 64$ counts are presented. 


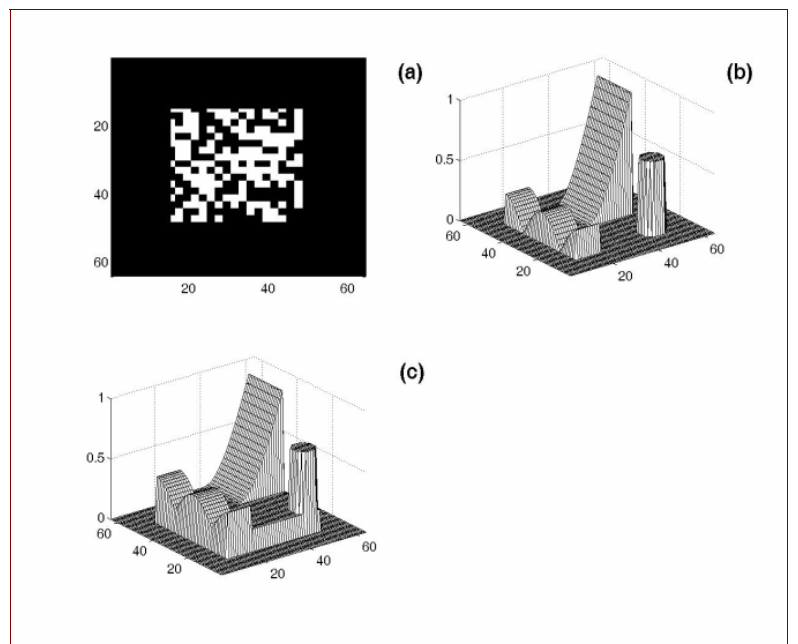

Fig. 2. Objects 64x64: (a) binary; (b), (c) half-tone without and with a base (equal to 0.17 )

\subsection{Binary object (the beam splitting)}

In Fig. 3, we present the plots characterizing the quality of the image of a binary object (Fig. 2a) reconstructed by a kinoform. As seen from Fig. 3a, the weighting algorithm allows one to decrease the dispersal of the one-bit-intensity $\Delta I_{\text {one-bit }}$ given by the ER-algorithm practically to zero, i.e., the algorithm does not reveal the effect of stagnation for binary objects. In our example, 180 weighting-iterations reduce $\Delta I_{\text {one-bit }}$ from 0.008 to $7.6 \times 10^{-7}$ (Fig. $3 \mathrm{~b})$, whereas 1500 such iterations result in $\Delta I_{\text {one-bit }}=2 \times 10^{-12}$. At the same time, the IO algorithm (with optimized $\mu$ ) "stops" at the value $\Delta I_{\text {one-bit }}=2.5 \times 10^{-5}$. That is, it falls in a minimum of $\sigma_{f}(k)$ which is sufficiently deep, but, nevertheless, is local. We note that the ratios of the minimum one-bit-intensity to the maximum zero-bit intensity for three algorithms are equal to, respectively, 4 (ER), 4.53 (IO), and 7 (weighting algorithm). Figure $3 \mathrm{~b}$ demonstrates the effect of a diminution of the variance $\sigma_{f}(k)$ at the transition from one algorithm to another one. Analogous results were obtained also for other binary objects with dimensions of $64 \times 64$ and $128 \times 128$.

\subsection{Half-tone object (the image generation)}

We observe a somewhat more complicated situation for half-tone objects, one of which is presented in Fig. 2b. As was shown by model experiments, the kinoforms of such objects calculated with the help of the weighting algorithm reconstruct a high-quality image only in the range of amplitudes from $\sim 0.15$ to 1 (at the normalization of the image to 1 ). The rest amplitudes are distorted to various degrees. We can reach the proper reconstruction of all amplitudes, including those close to zero, if the initial object is positioned on a pedestal (Fig. 2c), whose height is $\sim 15-20 \%$ of its maximum amplitude, and if the reconstructed image amplitude (the intensity in an optical experiment) is cut off by the pedestal level. It is obvious that, in this case, the useful diffraction efficiency of a kinoform decreases. The dependences of $\sigma_{f}(k)$ for both compared algorithms given in Fig. 4 , as well as the visual 

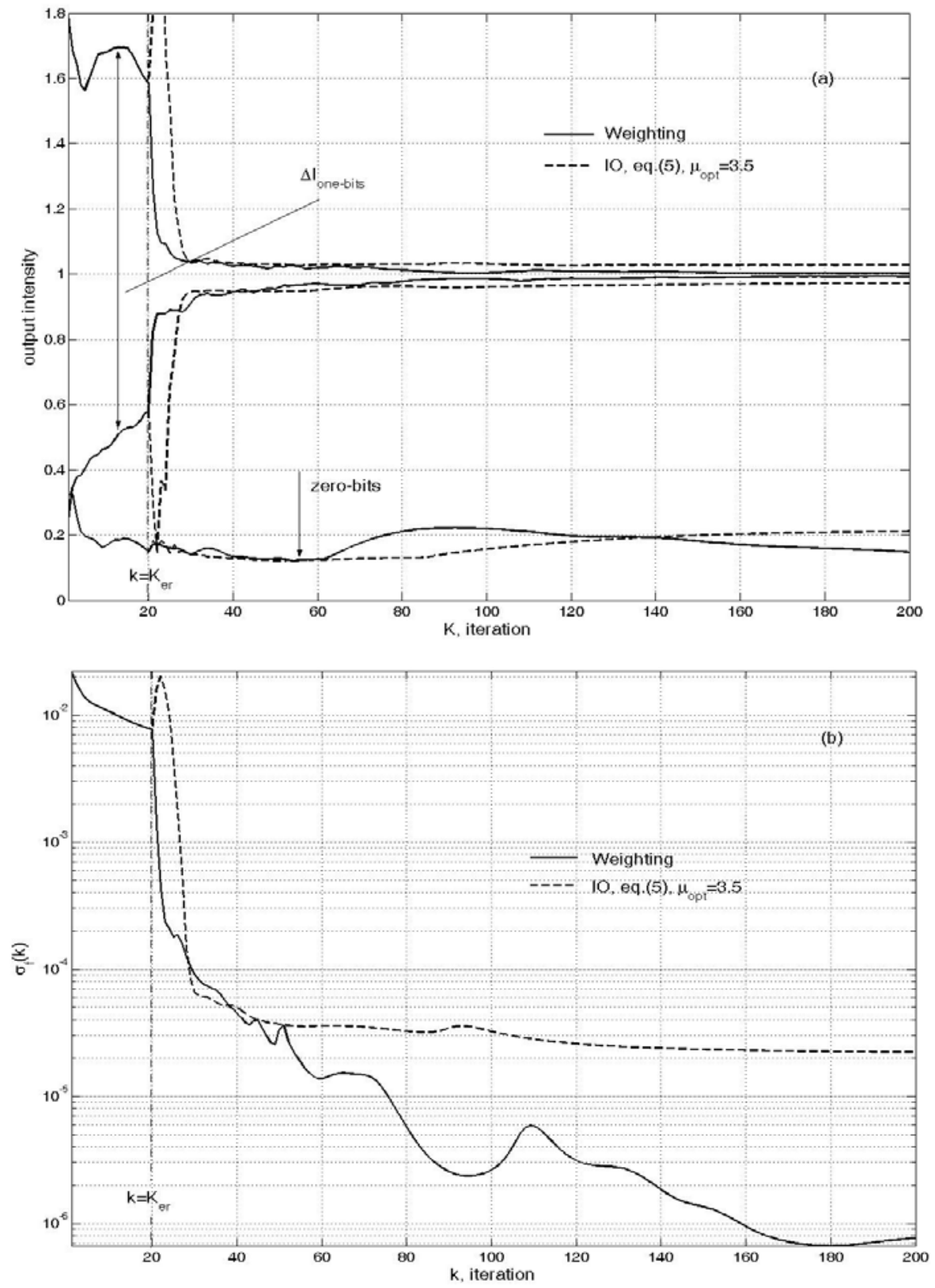

Fig. 3. The kinoform of the binary object (Fig. 2a): (a) range of output intensities, (b) variance of the amplitude of reconstructed image $v$ s the iteration number 
observations of reconstructed images, indicate that, in the case where a base is supplemented to an object, the weighting algorithm begins to surpass the IO algorithm in convergence after a certain number of iterations. In our example with the object in Fig. 2c, the advantage of the weighting algorithm over the IO algorithm begins to reveal itself after 100 iterations, increases with the number of iterations, and is almost four orders of magnitude by the 2000-th iteration $\left(5.8 \times 10^{-9}\right.$ against $2.5 \times 10^{-5}$ for $\left.\sigma_{f}(2000)\right)$. But if the base is absent, the IO algorithm has some advantage.

The calculated efficiencies of kinoforms (in parentheses, the values obtained within the IO algorithm are given) are as follows: 91.39 (91.25)\% for the object in Fig. 2a, 94.82 (92.48)\% for the object in Fig. 2b, and 96.91 (95.02)\% for the object in Fig. 2c. In the course of calculations, the criticality of the weighting algorithm with respect to a value of the parameter $\varepsilon$ in formula (4) is verified. By varying $\varepsilon$ from $1 \times 10^{-22}$ to $1 \times 10^{-6}$, the deviation of $\sigma_{f}(\varepsilon)$ from $\sigma_{f}\left(\varepsilon_{10}\right)=10^{-10}$ is determined as

$$
\Delta_{\sigma}=100 \%\left[\sigma_{f}(\varepsilon)-\sigma_{f}\left(\varepsilon_{10}\right)\right] / \sigma_{f}\left(\varepsilon_{10}\right)
$$

for various objects with the fixed number of iterations equal to 50 . On the average, $\Delta_{\sigma}$ was $(0.002-0.05) \%$. Thus, the variation of $\varepsilon$ in the indicated limits did not influence practically the exactness of the calculation of a kinoform and, at the same time, excluded the situation where one should divide the numerator in formula (4) by zero.

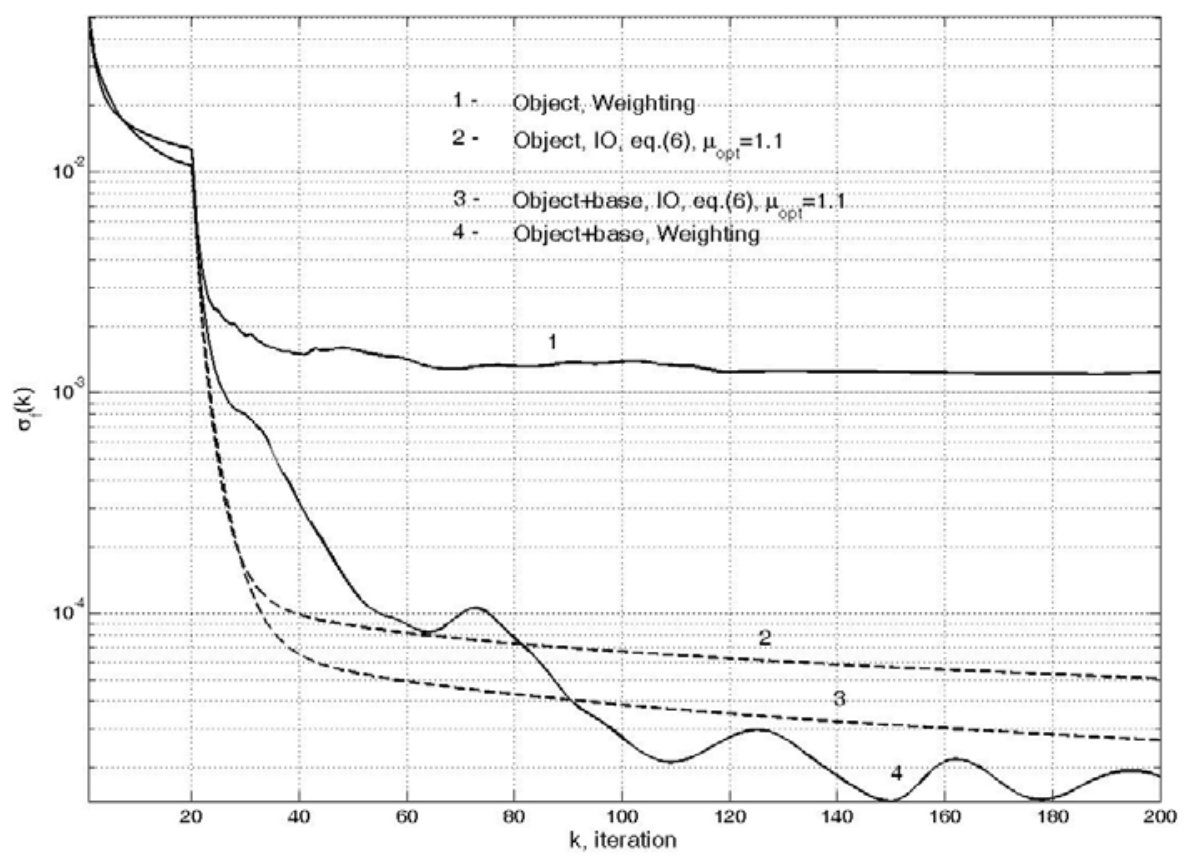

Fig. 4. Variance of the amplitude of reconstructed image for a half-tone object without and with a base (Fig. 2b, c) 


\subsection{Super-Gaussian (SG) beam shaping}

Within the weighting and IO algorithms, the calculations of kinoforms that are the transducers of the intensity of a Gauss beam of the form $\exp \left[-\left(u^{2}+v^{2}\right) / 2 r_{0}^{2}\right]$ in a SG beam of the form $\exp \left[-\left(x^{2} / 2 r_{o}{ }^{2}\right)^{M}-\left(y^{2} / 2 r_{o}{ }^{2}\right)^{M}\right]$ are performed, where $r_{o}$ and $r_{o}$ are the inflection radii of the Gauss curves, and $\mathrm{M}$ is the SG order (as known, the calculation of a kinoform involves the square root of the both indicated intensities). In Fig. 5, the input ( $r_{o}=$ $70)$ and output $\left(r_{o}^{\prime}=25\right)$ intensity profiles for $\mathrm{M}=4$ and $\mathrm{M}=100$ with a dimension of the object $256 \times 256$ counts are presented. The iteration process with $K_{e r}=10$ was truncated at the 100-th iteration. With regard for the separation of the working part of a SG beam so as shown in Fig. 5, the intensity variance $\sigma_{I}$ and the output efficiency $\eta$ are as follows: $\sigma_{I}=2.9 \times 10^{-4}\left(6.59 \times 10^{-4}\right), \quad \eta=96.46(89.72) \%$ for $\mathrm{M}=4 ; \sigma_{I}=3.7 \times 10^{-5}\left(1.98 \times 10^{-4}\right)$ ， $\eta=93.63(91.2) \%$ for $\mathrm{M}=100$. The calculation of $\sigma_{I}$ was performed by a form analogous to (4), but for intensities. It should be noted that, in the calculation of a kinoform-former of a SG, a special attention should be paid to a choice of $r_{o}$ defining the effective width of a beam illuminating the kinoform. For small $r_{o}$ (in our example, $r_{o}=35$ ), the kinoform is illuminated by a narrow Gauss beam, which means the actual nullification of light amplitudes on the edges of the kinoform. This is equivalent to a reduction of the band of space frequencies forming a SG. As a result, the pattern of a SG will be covered by a speckle irrespective of the value of $r_{o}^{\prime}$ (see Fig. 6). In more details, the problem of restriction of the frequency band and its relation to the quality of images are considered formerly (Wyrowski and Bryngdahl, 1988).

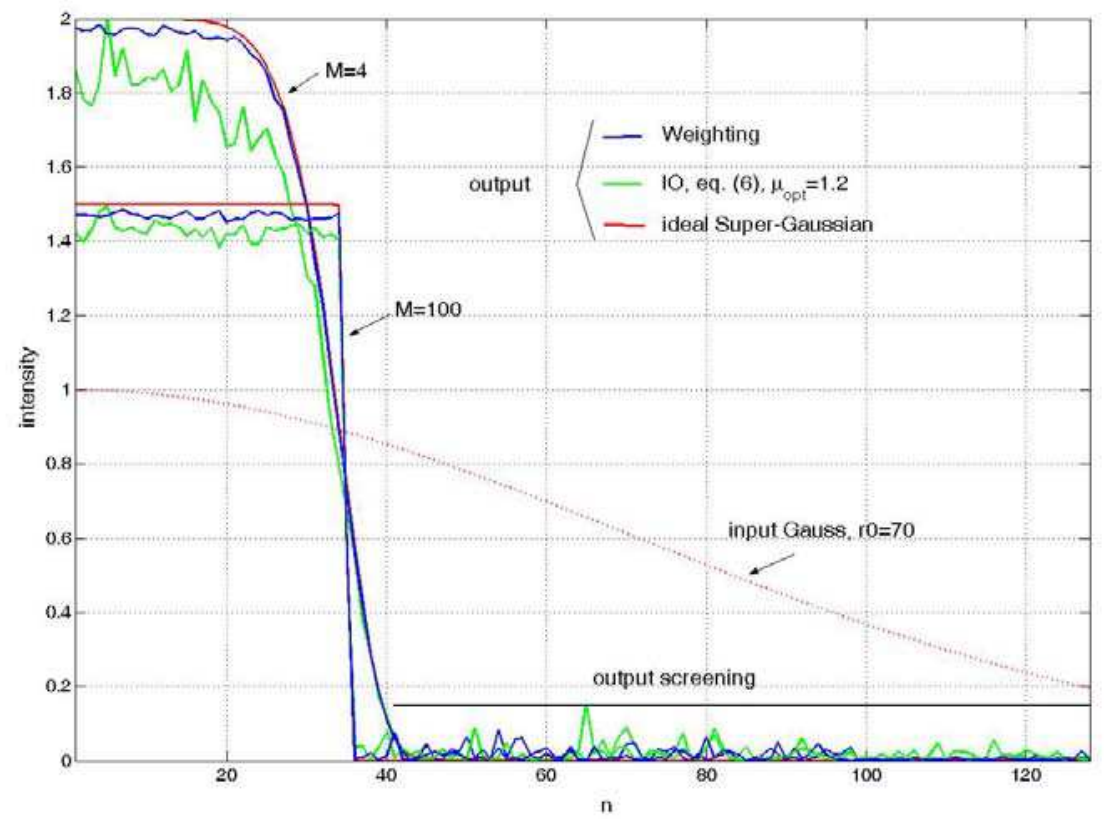

Fig. 5. The profiles of intensities of super-Gaussian beams with $r_{o}^{\prime}=25$ of the $4^{\text {th }}$ and $100^{\text {th }}$ orders within the weighting and IO algorithms. Curves for $M=4$ and $M=100$ are vertically shifted up for clarity 


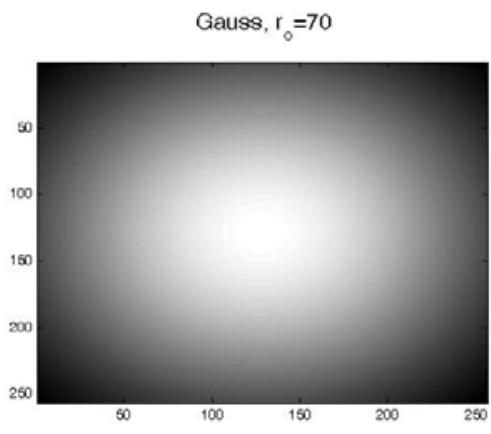

Gauss, $r_{0}=35$

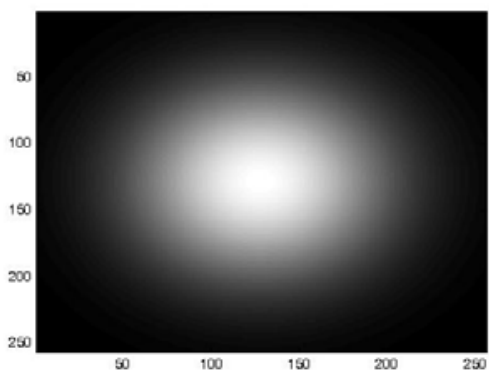

$S G, r_{0}=25$

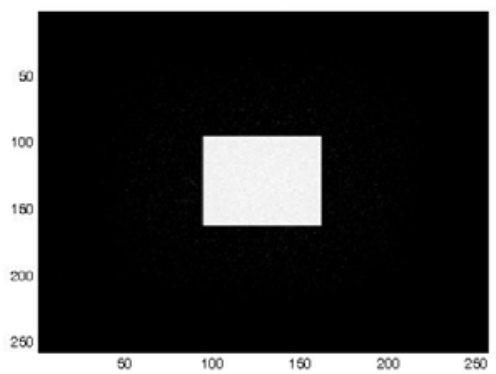

SG, $r_{0}=25$

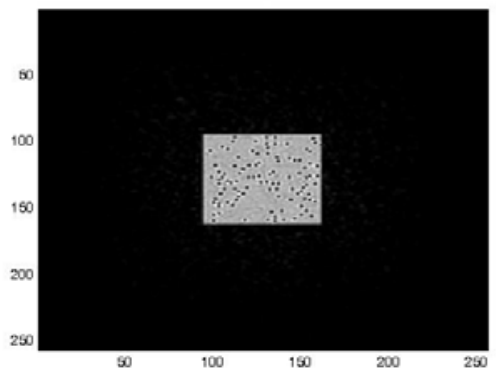

Fig. 6. Proper $\left(r_{o}=70\right)$ and erroneous $\left(r_{o}=35\right)$ choices of the effective width of an illuminating beam for the kinoform-former of a super-Gaussian $\left(r_{o}^{\prime}=25\right)$. In the second case, the cross-section of a super-Gaussian is covered by a speckle

\subsection{Off-axis kinoform}

Irrespective of the mean and the accuracy of calculations of the phase function $\psi$ of a kinoform, the quality of a reconstructed image depends eventually on the accuracy of the representation of a microrelief of this phase on a recording medium. It is obvious that this accuracy depends on the technical potentialities of a registering unit and the characteristics of a recording medium. As for a programmed SLM, the accuracy is determined by its physico-technical parameters. In the synthesis of an axial Fourier-kinoform which reconstructs the image in the zero order of diffraction, the inaccuracy of the representation of the phase $\psi$ leads to the appearance of a bright spot surrounded by noises at the center of the image. This effect can be eliminated in the single way due to a displacement of the image, as a whole, to the side from the optical axis of a reconstruction system. This can be achieved by the synthesis of a kinoform which reconstructs the image in the nonzero order. Such a kinoform is called the off-axis one.

One knows the mean of synthesis (S1) of an off-axis kinoform (Wyrowski, 1990) with the reconstruction of the image in a nonzero order with components $\mathrm{P}_{\mathrm{x}}, \mathrm{P}_{\mathrm{y}}$ along the axes $x, y$ of the plane of images. The components $\mathrm{P}_{\mathrm{x}}$ and $\mathrm{P}_{\mathrm{y}}$ are defined identically. Therefore, in what follows, we will write all relations only for the axis $x$. The admissible linear displacement $x_{0}$ of an image from the optical axis of the Fourier-system of reconstruction in mean S1 is defined as 


$$
x_{0}=P_{x} L^{p}
$$

where

$$
P_{x} \leq \frac{1}{2}\left(1-\frac{L^{i}}{L^{p}}\right),
$$

$L^{i}$ - linear sizes of the image along the axis $x$, and $L^{p}$ - linear sizes of the diffraction order. According to (10) and (11), the value of $x_{0}$ decreases with increase in $L^{i}$. At the standard values $L^{i} \approx L^{p} / 2$, we get $0 \leq P_{x} \leq 1 / 4$. Respectively, the image can be displaced in the interval $0 \leq x_{o} \leq L^{p} / 4$. From the viewpoint of practice, the principal drawbacks of mean S1 are both a small interval of admissible displacements of the image and the dependence on the image size. As a positive feature of the mean, we mention the invariance of the total number of pixels of a kinoform at the transition from the axial to off-axis variant of the synthesis.

One knows also the mean of synthesis (S2) of an off-axis kinoform (Turunen et al., 1990) with the possibility of the reconstruction of an image in the order $P_{x} \neq 0$ which can vary in the limits $0 \leq P_{x} \leq 1$ for the same limitations on $\mathrm{L}^{\mathrm{i}}$ as in mean S1. Mean S2 ensures a wide interval of displacements $x_{o}\left(0 \leq x_{o} \leq L^{p}\right)$ which does not depend on the image size. However, this is attained due to the increase in the total number of pixels necessary for the registration of a kinoform by $K$ times (practically, $2 \leq K \leq 8$ ). The calculation and the registration of such kinoforms (e.g., by the methods of laser or electron lithography) are quite complicated (Turunen et al., 1990). The use of programmed SLMs (the total number of pixels is $\approx 10^{3} \times 10^{3}$ on the average) for their representation becomes problematic already for the dimension of a kinoform of $256 \times 256$ and $K>4$.

We propose a mean of synthesis of an off-axis kinoform which ensures a significantly greater interval of admissible displacements of the reconstructed image as compared with mean S1. In this case, we conserve the main advantage of the latter, namely the invariability of the total number of pixels of a kinoform at the transition from the axial to off-axis variant of the synthesis. The essence of the mean is simple: in order to make a kinoform to be offaxis, we have to introduce the spatial carrier frequency to it. To this end, we propose to supplement of any IFT-method of calculation of the kinoform (including weighting algorithm) by one more operation - to add the linear phase $2 \pi\left(x_{0} u+y_{0} v\right)\left(x_{0}, y_{0} \geq 0\right.$ or $\left.\leq 0\right)$ to the phase $\psi(u, v)$ of an on-axis kinoform at the last iteration. As a result, the calculated kinoform will reconstruct the image

$$
\begin{aligned}
f_{\text {off }}(x, y) & =\mathfrak{J}^{-1}\left\{\exp \left[i\left(\psi(u, v)+2 \pi\left(x_{o} u+y_{o} v\right)\right]\right\}=\right. \\
& =f(x, y) \otimes \delta\left(x-x_{o}, y-y_{o}\right)=f\left(x-x_{o}, y-y_{o}\right) .
\end{aligned}
$$

Here, $f_{\text {off }}(x, y)$ - off-axis image, $f(x, y)$ - axial image, $\delta$ - delta-function, and the symbol $\otimes$ stands for the operation of convolution. It follows from (12) that $f_{\text {off }}$ is nothing but the axial image displaced along the axes $x, y$ by $x_{0}, y_{0}$. The values of $x_{0}, y_{0}$ (and also the order $\mathrm{P}_{\mathrm{x}}, \mathrm{P}_{\mathrm{y}}$ ) are independent of the ratio $\mathrm{L}^{\mathrm{i}} / \mathrm{LP}$ in this case, as distinct from mean S1, and can be, in principle, arbitrary. However, the optical and model experiments executed by us have shown that $x_{0}, y_{0}$ should be chosen in the limits $0 \leq x_{0}, y_{0} \leq L^{p} / 2$, which corresponds to 
$0 \leq P_{x}, P_{y} \leq 1 / 2$. Thus, the image can be displaced in the proposed mean in the interval $0 \leq x_{0}, y_{0} \leq L^{p} / 2$ which exceeds at least twice the analogous interval in mean S1. In Fig. 7a, we show the example of the reconstruction into a fractional order $\left(P_{x}=0.45, P_{y}=0\right)$ (capture of $3 \times 3$ diffraction orders). The off-axis kinoform was calculated using the weighting IFTalgorithm with the introduction of a spatial carrier frequency (a deflecting grating) along the axis $x$. In Fig. 7b, we give a fragment of the cross-section of a given grating. An analogous complicated structure of the grating is observed for the remaining values of $\mathrm{P}_{\mathrm{x}}, \mathrm{P}_{\mathrm{y}}$ except for $\mathrm{P}_{\mathrm{x}}, \mathrm{P}_{\mathrm{y}}=0.25$ and 0.5 (for them, the grating periods are, respectively, $0, \pi / 2, \pi, 3 \pi / 2$, and $0, \pi)$.

It is obvious that, in order that such complicated grating have a sufficient number of periods $T_{g}$ of oscillations in the structure of a kinoform (and thus could manifest the deflecting properties), the kinoform format must be sufficiently great ( $\geq 500 \times 500$ pixels). It is worth noting that the diffraction efficiency of an off-axis kinoform decreases with increase in a displacement of the image. In Section 4, we present the quantitative results of measurements of the diffraction efficiency of off-axis kinoforms.

\subsection{Iterative quantization}

For practical reasons, the phase structure of a kinoform $\psi(u, v)$ is usually quantized. This simplifies the production step. We investigated both the direct iterative quantization and the stepwise (soft) iterative quantization. The former is given by the standard operator

$$
\bar{\psi}(u, v)=\left\{\begin{array}{cc}
0, & \psi(u, v) \leq 0.5 \Delta \psi \\
\vdots & \vdots \\
m \Delta \psi, & (m-0.5) \Delta \psi \leq \psi(u, v) \leq(m+0.5) \Delta \psi \\
\vdots & \vdots \\
2 \pi, & \psi(u, v) \geq(M-0.5) \Delta \psi
\end{array}\right.
$$

and the latter is presented by the operator (Wyrowski, 1990)

$$
\bar{\psi}(u, v)=\left\{\begin{array}{cc}
0, & \psi(u, v) \leq 0.5 \varepsilon^{(p)} \Delta \psi \\
\vdots & \vdots \\
m \Delta \psi, & \left(m-0.5 \varepsilon^{(p)}\right) \Delta \psi \leq \psi(u, v) \leq\left(m+0.5 \varepsilon^{(p)}\right) \Delta \psi \\
\vdots & \vdots \\
2 \pi, & \psi(u, v) \geq\left(M-0.5 \varepsilon^{(p)}\right) \Delta \psi \\
\psi(u, v), & \text { otherwise }
\end{array}\right.
$$

where

$$
\begin{aligned}
& m=0,1, \cdots, M, \Delta \psi=2 \pi / M, M-\text { number of quantization levels, } \\
& 0<\varepsilon^{(1)}<\varepsilon^{(2)}<\cdots \varepsilon^{(p)} \cdots<\varepsilon^{(P)}=1, \\
& p=1,2, \cdots P, P-\text { number of stages of quantization. }
\end{aligned}
$$

The principle of stepwise quantization consists in the following. The whole process of iteration is divided into $P$ cycles, each of which (except for the last one) includes $Q$ iterations. In the course of the implementation of a cycle, only 

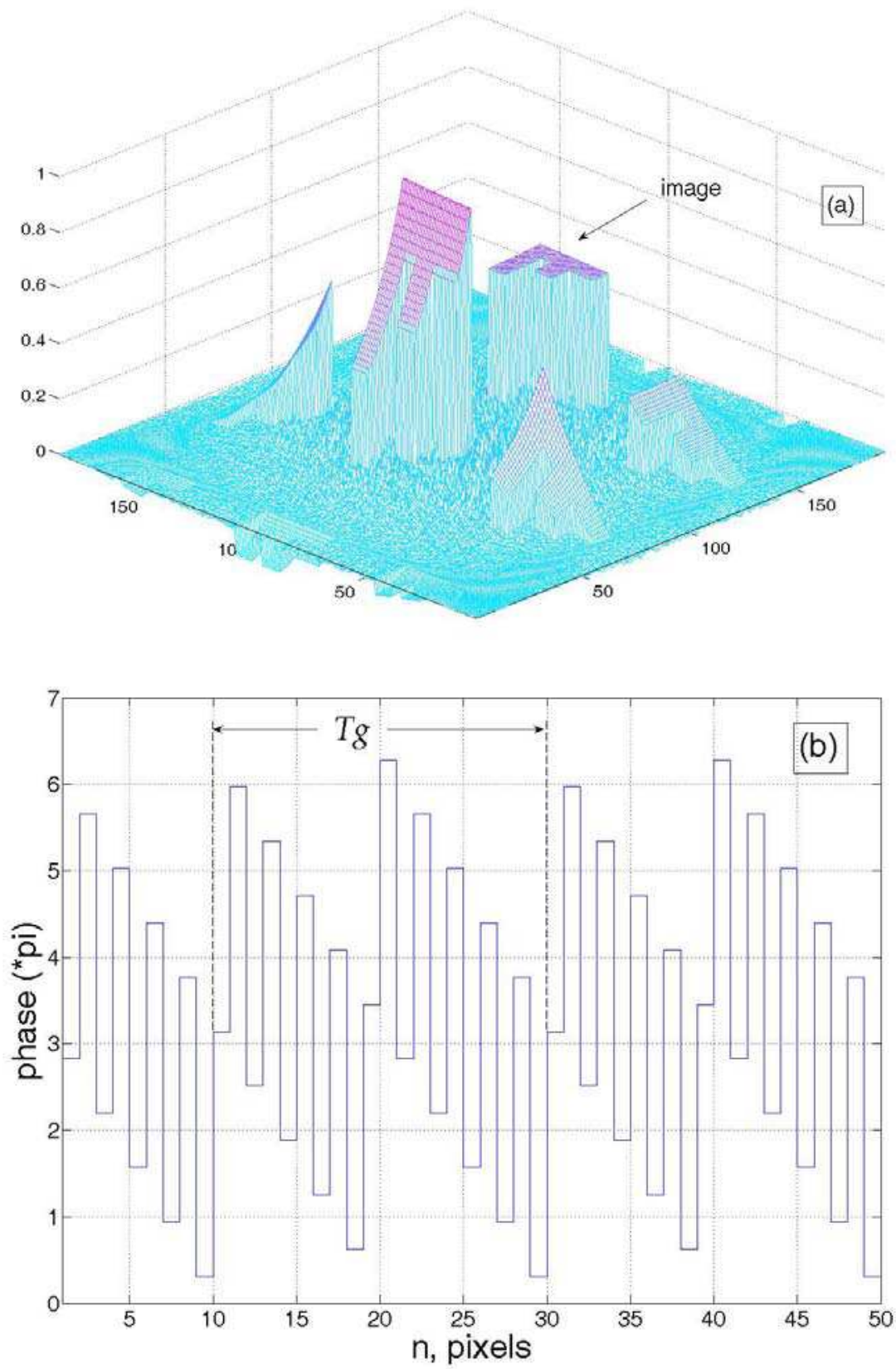

Fig. 7. (a) reconstruction into the order $P_{x}=0.45, P_{y}=0$; (b) fragment of the spatial carrier frequency 
a part of the phases of a kinoform, which belong to the interval $\mathcal{E}^{(p)} \Delta \psi<\Delta \psi$ is quantized, rather than all phases falling in the interval $\Delta \psi$ (relate to some $m$-level of quantization). The remaining phases remain invariable. The quantity $\varepsilon$ increases with the index $p$. Respectively, the interval of quantized phases is extended, by attaining eventually a value close to $\Delta \psi$. At the last step $(P)$, the direct quantization operator is reached, and only one iteration is performed. Thus, the total number of iterations $K=Q(P-1)+1$. Values of $P$ and $Q$ can be, in principle, arbitrary, as well as the values of elements of the sequence $\varepsilon^{(p)}$. Their optimization is attained experimentally. Some versions of the choice of $Q, P$ and $\varepsilon$ for $K=$ const were considered by Skeren et al. (2002). But we used, in our experiments with binary objects, the collection of values

$$
\begin{aligned}
& P=10, \\
& \varepsilon^{(1)}=0.3, \varepsilon^{(2)}=0.5, \varepsilon^{(3)}=0.6, \varepsilon^{(4)}=0.7, \varepsilon^{(5)}=0.75, \\
& \varepsilon^{(6)}=0.8, \varepsilon^{(7)}=0.85, \varepsilon^{(8)}=0.9, \varepsilon^{(9)}=0.95, \varepsilon^{(10)}=1,
\end{aligned}
$$

which was proposed and approved by Wyrowski (1990) and allows one to improve, in dependence on the number of quantization levels and the form of an object, the signal/noise ratio for the reconstructed image by 3 to 10 times as compared with that for the direct quantization.

\section{Experiment}

\subsection{Optical-digital system}

Model experiments (see Section 3) have shown the high efficiency of the weighting IFTalgorithm just for binary objects. Therefore, we investigated kinoforms acting as a beam splitter and the generation of patterns of binary objects. A typical optical-digital Fourier system (Fig. 8) with a He-Ne laser $(543 \mathrm{~nm})$ is used to investigate the kinoform reconstruction characteristics. Here, we use a reflection-type phase-only SLM HEO 1080 Pluto produced by the HOLOEYE Inc. The reconstructed images were recorded and processed with the use of a SP620-USB CCD-camera of Spiricon Inc. with a high dynamic range.

As known (Oton et al., 2007), the spatial calibration of reflective LCoS SLM is essential for the correct use of the modulator in applications with high requirements of the wavefront control. We determined the additional phase 2D-distribution compensating the distortions of a wave front which appear due to the imperfection of SLM (backplane curvature, thickness variations of the liquid crystal layer across the aperture of the SLM, and so on) and elements of the optical system, by using the interference-based method proposed by Oton et al. (2007). At the implementation of experiments, we sum the obtained distribution with the calculated phase of a kinoform. This allowed us to exclude, to a significant degree, the hardware-based effect at the measurement of characteristics of reconstructed images. The size of objects and kinoforms was 1000×1000 pixels in format. As objects, we took letter F and a $14 \times 14$ two-dimensional one-bits array occupying, respectively, $250 \times 150$ and $200 \times$ 200 counts of the input plane which are used in the study of the output intensities, diffraction efficiency, and effects of quantization of the kinoform phase in the reconstructed images. 
Weighting Iterative Fourier Transform Algorithm for

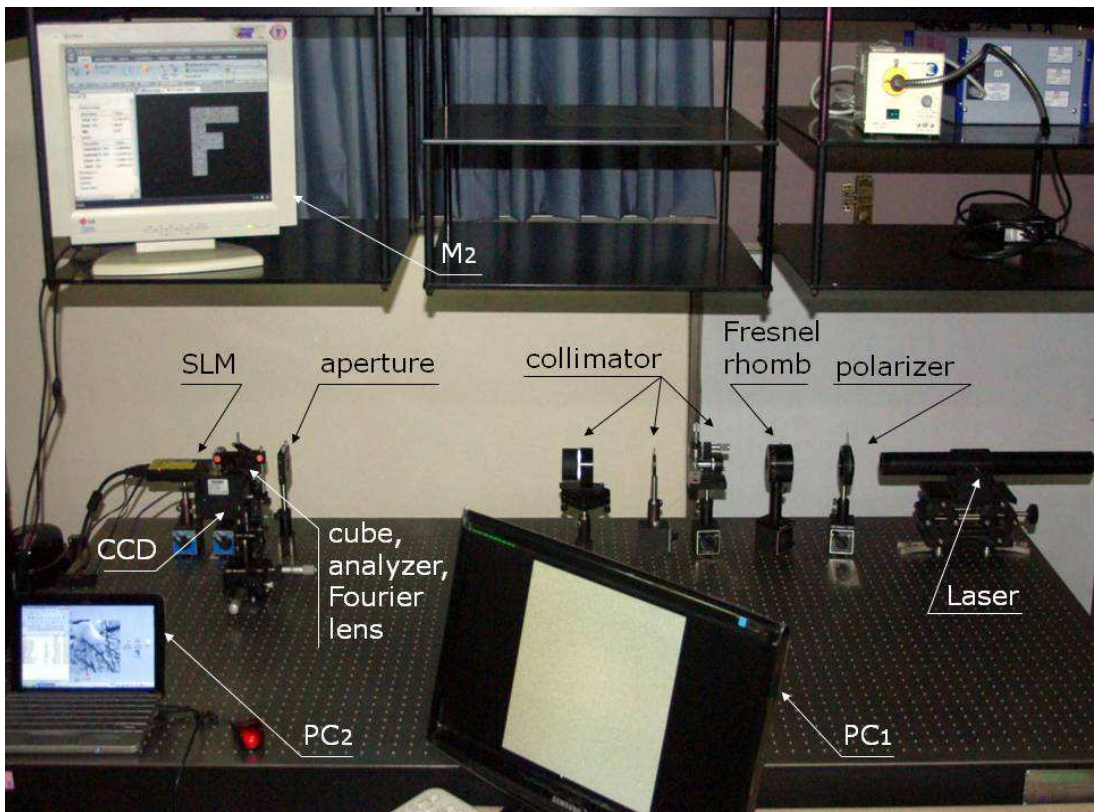

Fig. 8. Optical-digital Fourier system. Notations: SLM - SLM HEO 1080 PLUTO, CCD SPU620 CCD with BeamGage software, PC1, PC2+M2 - computers for the control, respectively, SLM and CCD-camera, Laser - He-Ne laser (543 nm)

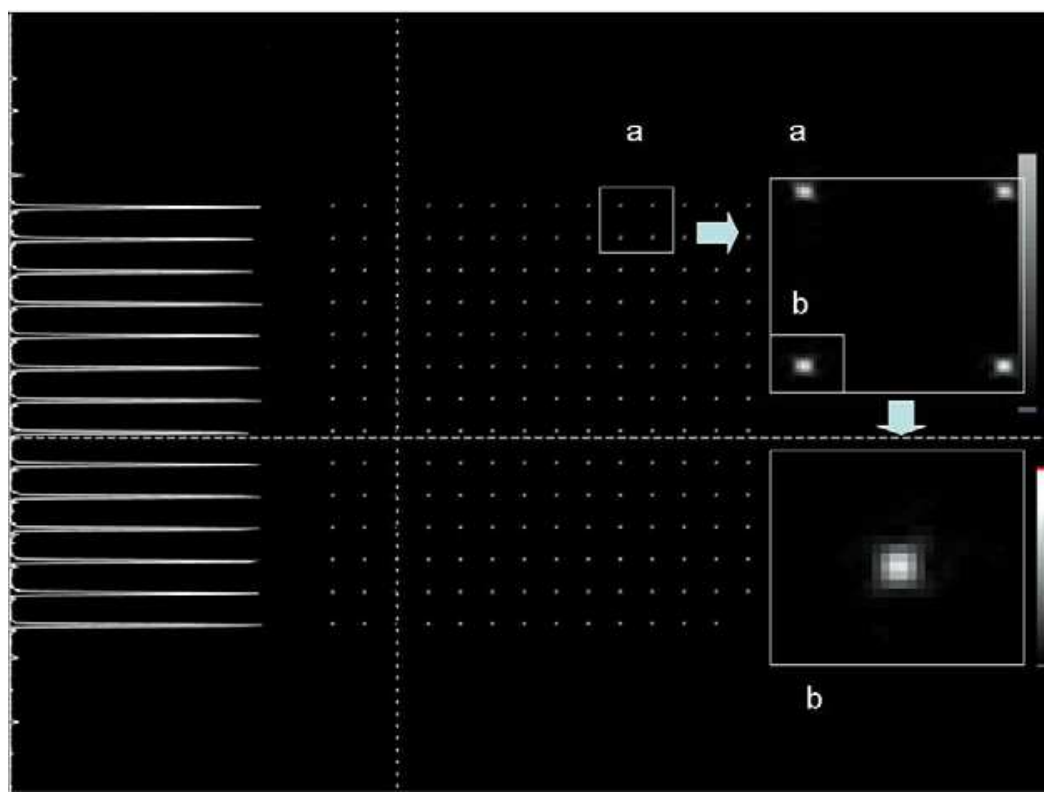

Fig. 9. Kinoform as a beam-splitter: reconstructed image of $14 \times 14$ spots with the intensity profile (third column) and the structure $(a, b)$ of spots 


\subsection{Kinoform as a beam-splitter}

In Fig. 9, we present the results of experiments with the use of a kinoform for the twodimensional spot array generation. In calculations, we applied the stepwise quantization with parameters given in relation (16), the number of quantization levels $M=256$, and the ratio of iterations ER/weighting $=10 / 15$. The measured profile of intensities demonstrates a high homogeneity of light spots. Each spot of an image was registered by an area including $9 \times 9$ pixels of a CCD-camera, which allows us to control the regularity of arrangement of intensity maxima of spots in the output plane. No deviations from the regularity were observed.
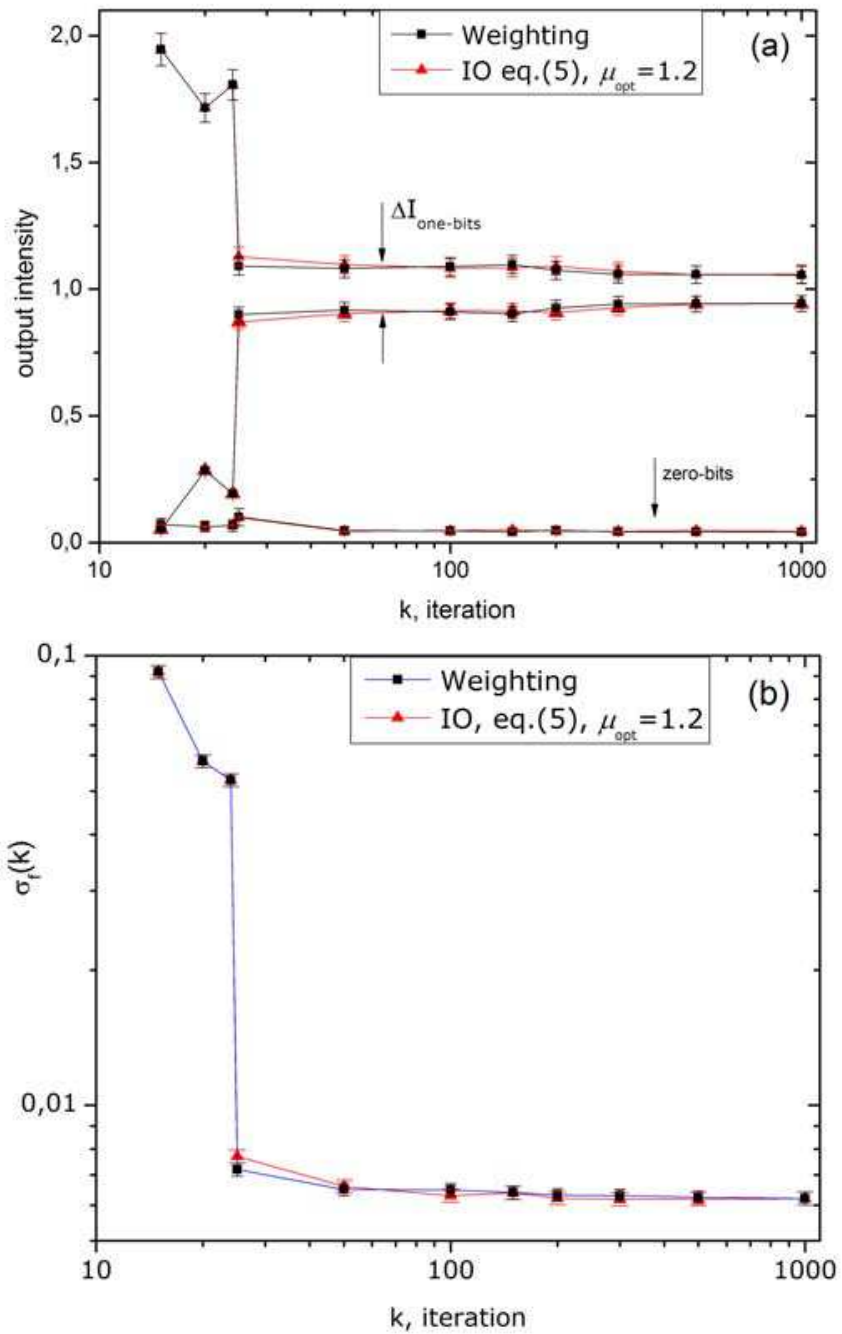

Fig. 10. Kinoform as a beam-splitter: (a) range of output intensities, and (b) variance of spots - intensities $v s$ the iteration number 


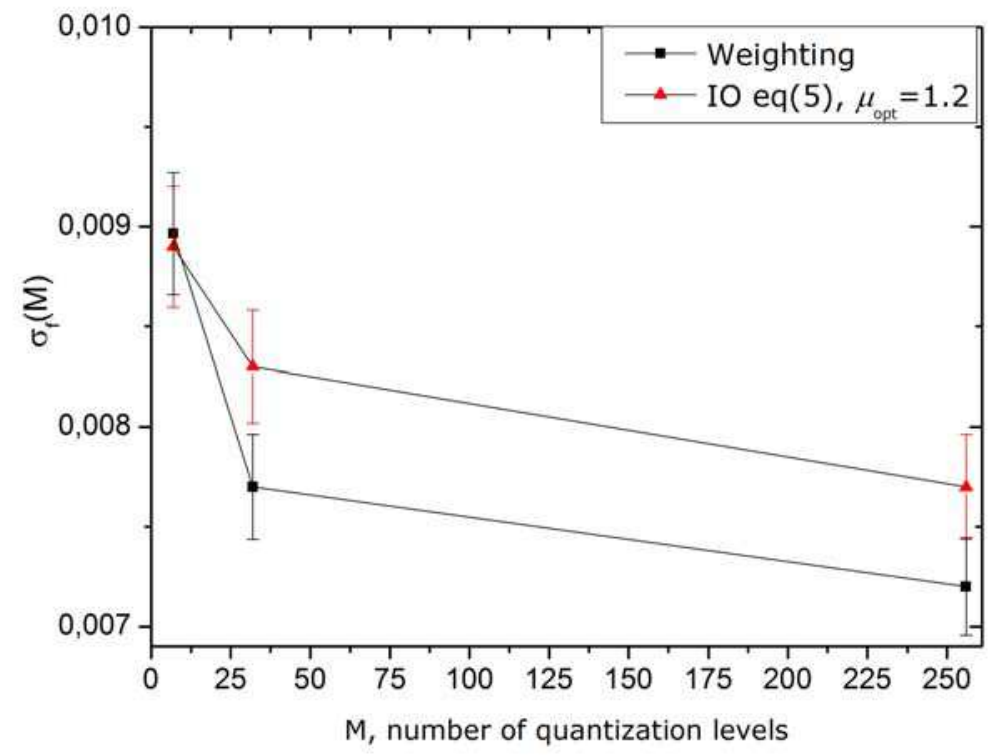

Fig. 11. Kinoform as a beam-splitter: variance of a reconstructed image $v s$ the number of quantization levels of the kinoform phase

In Fig. 10, the plots characterizing the quality of the image (Fig. 9) reconstructed by a kinoform are presented. As seen, both methods give practically the same experimental results as distinct from the model experiments with binary objects (see Fig. 3), in which the weighting method has had the obvious advantage over the IO method. The reasons for this situation will be discussed in Conclusion in more details. In Fig. 11, we show the variance of a reconstructed image as a function of the number of quantization levels. In the obtaining of curves in Figs. 10 and 11, kinoforms calculation was performed with use the stepwise quantization at the ratio of iterations ER/weighting=10/15.

\subsection{Off-axis kinoform}

In Figs. 12 and 13, we give the results of experiments with off-axis kinoforms for object-letter F. Figure 12 demonstrates the example of the reconstruction into partial orders $P_{x}=0,0.25$, and 0.50 . As expected, the $0^{\text {th }}$ and $1^{\text {st }}$ orders remain immobile, and the image shifts between them. It is seen from Fig. 13 how the diffraction efficiency (DE) of a kinoform varies at the successive shift of the image. Curve 1a corresponds to a shift along the axis $X$, and curve $2 \mathrm{a}$ does to a shift along the diagonal in the $X, Y$-plane $\left(P_{x}, P_{y}=0,0.1,0.15, \ldots, 0.50\right)$. In this case, the synthesis of kinoforms is realized with the help of the weighting algorithm at the ratio of the numbers of iterations ER/Weighting $=20 / 200$. Curves $1 b$ and $2 b$ represent analogous dependences, but for the synthesis of a kinoform with addition of amplitude freedom iterations (Wyrowski, 1990). While applying the amplitude freedom, the zero-noise on the format of a valid image decreases practically to zero, however, the DE decreases significantly in this case. In the second case, the ratio of iterations ER/Weighting/Amplitude freedom $=20 / 50 / 150$. In Table 1, we present the more detailed 
quantitative data. In measurements, we used a Newport Dual Channel Power meter, Model No. 2832-C. The error of measurements was $2.3 \%$ on the average. A decrease in DE at a shift if the image is explained by the finite size of the SLM pixel aperture, in the meanwhile the character of this decrease does not depend on the method of calculation of a kinoform. The synthesis within the input-output method gives close results. The aperture distortion of an image is compensated by multiplication, at the beginning of iterations, of the input function of an object $f_{o}$ by the inverse function of the pixel aperture $\sin c^{-1}$ (Lohmann \& Paris, 1967; Kuzmenko \& Yezhov, 2007). We note that the upper bound of DE for object-letter F, covered by the optimized scatterer, calculated by the method Wyrowski (1991) is $90.36 \%$. Thus, the energy losses due to the presence of the zero and higher orders of diffraction are, in the best case (curve $1 \mathrm{a}, \mathrm{Px}=0$ ) of the order of $\sim 27 \%$.
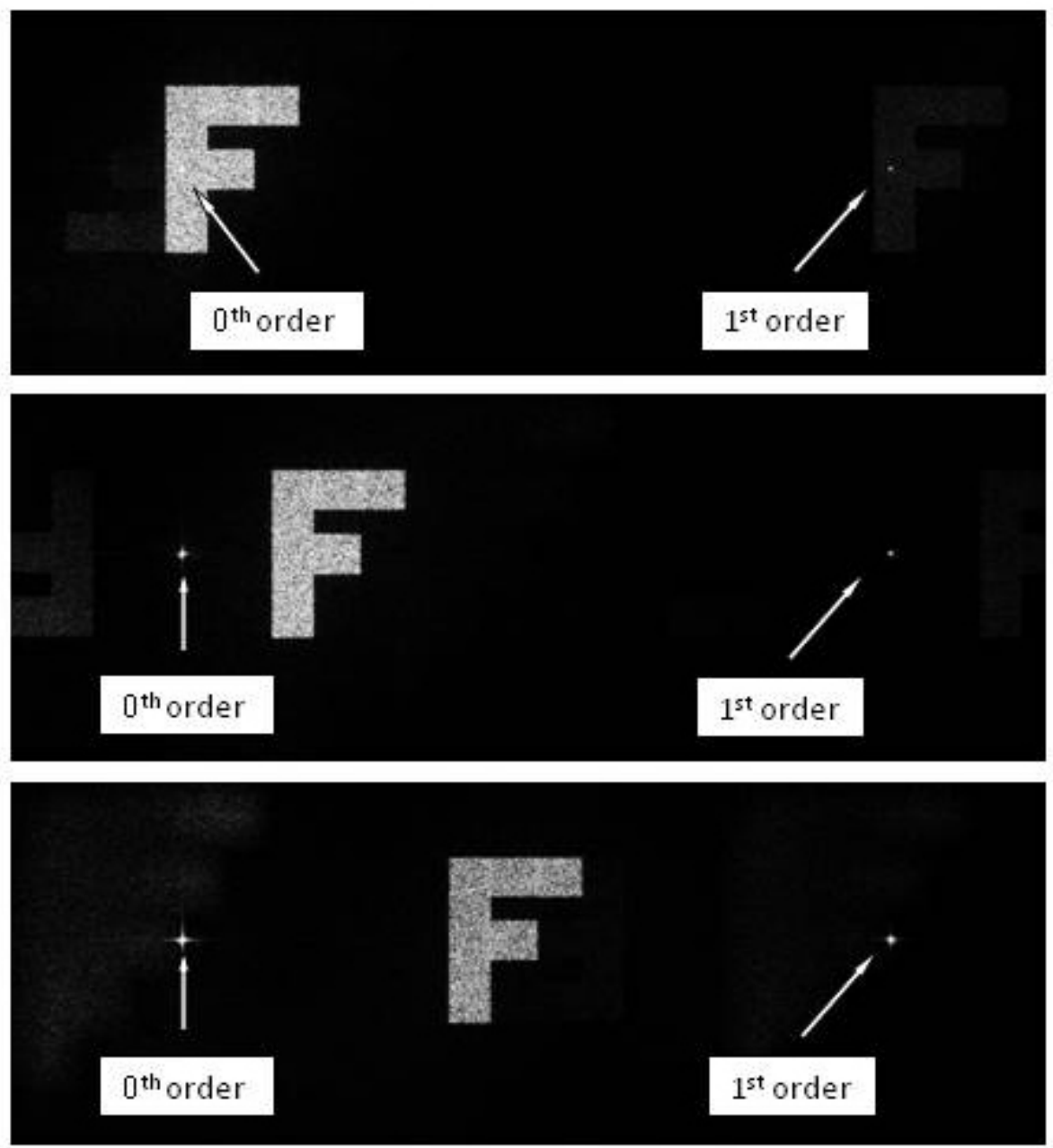

Fig. 12. Off-axis kinoform. Reconstruction into partial diffraction orders, $P_{x}=0,0.25$, and 0.5 


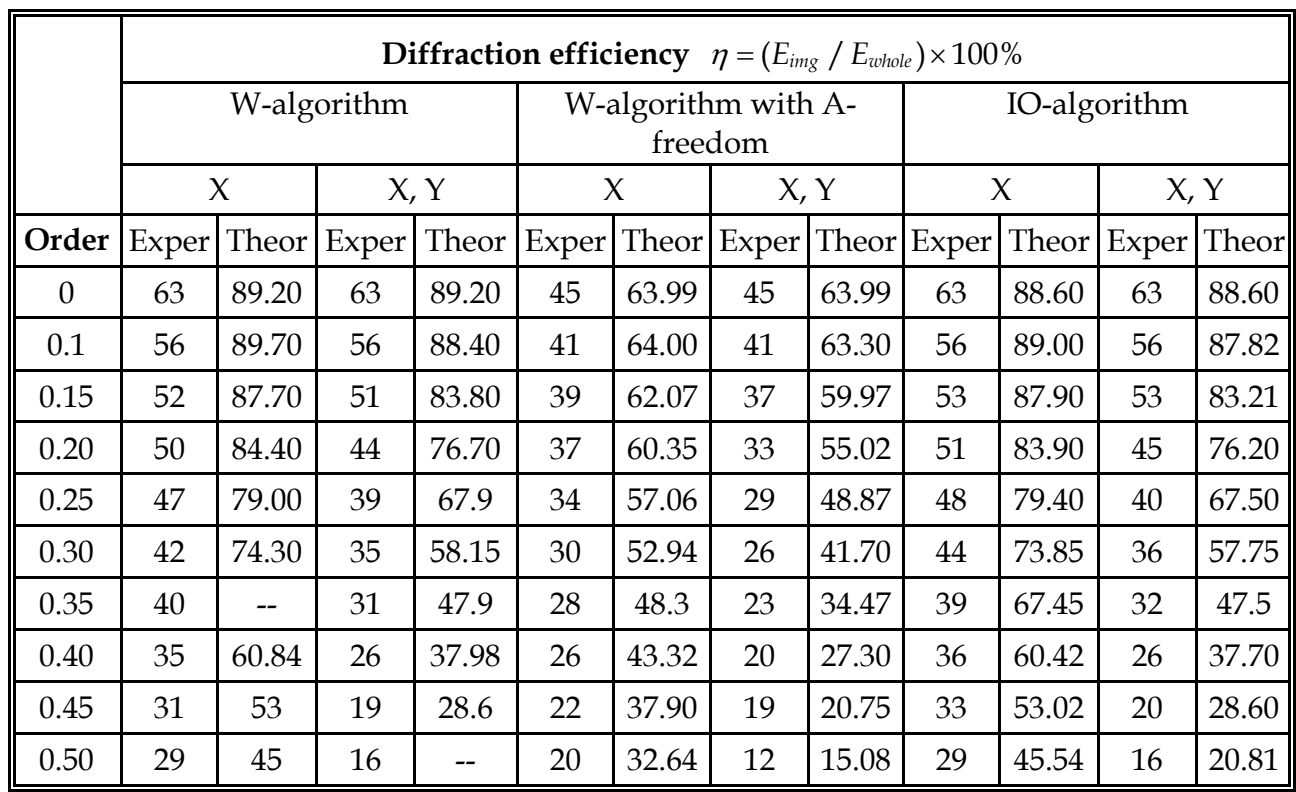

Table 1. Diffraction efficiency vs the partial diffraction order. $X$ - means the image in a partial order along the axis $X ; X, Y-$ means the image in a partial order along the diagonal of the $X-Y$ plane. A-freedom means the use of amplitude-freedom iterations at the final stage of calculations. The relative error of measurements $\Delta \eta / \eta \approx 2.3 \%$

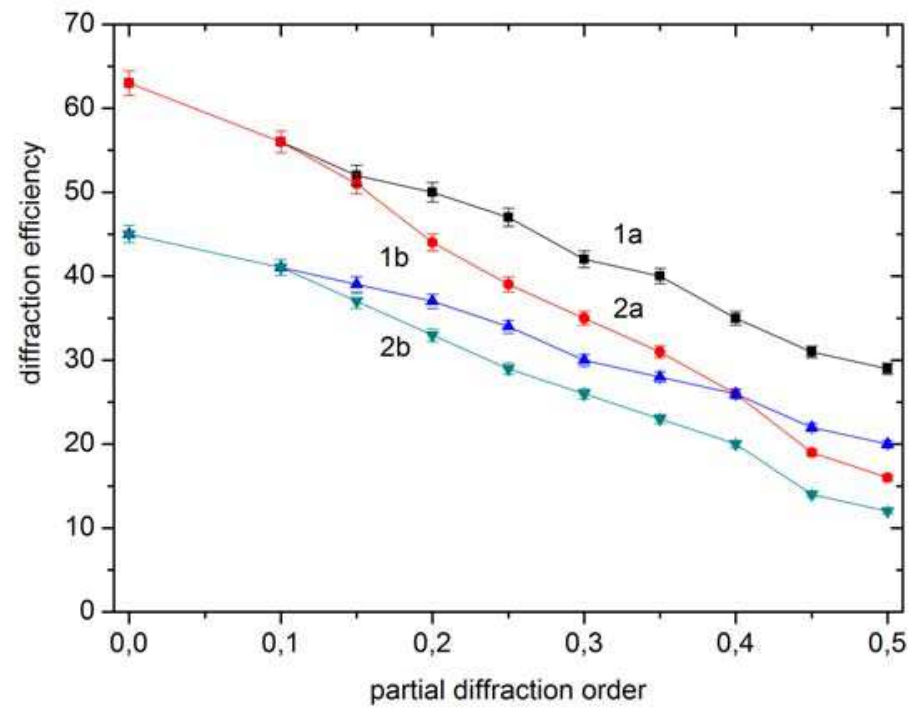

Fig. 13. Diffraction efficiency of the off-axis kinoform $v$ s the partial diffraction order (for object-letter F) 


\section{Conclusion}

Summarizing, we may assert that the weighting algorithm has high efficiency in the synthesis of the kinoforms of binary objects. It is worth noting that the effect of stagnation of the algorithm is absent in this case, i.e., the one-bits variance $\sigma_{f}(k)$ in a reconstructed image tends to zero with increase in the number of iterations, and the noise level (zero-bits) is the same as that of other algorithms. The weighting algorithm is also efficient in calculations of kinoforms as the formers of super-gaussian laser beams. It must be emphasized that the weighting algorithm contains no parameters requiring the optimization (like the feedback parameter $\mu$ in the IO algorithm), which essentially accelerates the counting rate.

However, as noted in the literature (Skeren et al., 2002), the methods of synthesis of kinoforms, which differ in accuracy, can give practically identical results. The matter is in that the physico-technical parameters of the available SLM do not allow one to completely realize the potentialities of high-accuracy algorithms. This is indicated by the abovepresented results of experiments, where the comparison of the weighting and IO methods is performed. It is possible to assert that all algorithms ensuring $\sigma_{f}(k) \approx 1 \times 10^{-4}$ or less at the realization of a kinoform on SLM of the type used by us give images of the approximately identical quality.

\section{References}

Aagebal, H. \& Wyrowski, F. (1997). Paraxial beam splitting and shaping. In Diffractive Optics for Industrial and Commercial Applications, J. Turunen and F. Wyrowski, ed. (Academie Verlag, Berlin 1997), Chapter 6, pp. 165-188.

Akahori, H. (1986). Spectrum leveling by an iterative algorithm with a dummy area for synthesizing the kinoform. Appl. Opt., vol. 25, pp. 802-811.

Bryngdahl, O. \& Wyrowski, F. (1990). Digital holography - computer-generated holograms. In Progress in Optics, E. Wolf, ed. (North-Holland, Amsterdam, 1990), vol. 28, pp. 186.

Catino, W. C.; LoCicero J. L. \& Stark H. (1997). Design of continuous and quantized phase holograms by generalized projections. JOSA A, vol. 14, pp. 2715-2725.

Chen, W.; Roychoudhuri C. S. \& Banas C. M. (1994). Design approaches for laser-diode material-processing systems using fibers and micro-optics. Opt. Eng., vol. 33, pp. 3662-3669.

Dixit, S. N.; Lawson J. K.; Manes K. R.; Powell H. T. \& Nugent K. A. (1994). Kinoform phase plates for focal plane irradiance profile control. Opt. Lett., vol. 19, pp. 417-419.

Duparre, M.; Golub M. A.; Ludge B.; Pavelyev V. S.; Soifer V. A. \& Uspleniev G. V. (1995). Investigation of computer-generated diffraction beam shapers for flattening of single-modal $\mathrm{CO}_{2}$ laser beams. Appl. Opt., vol. 34, pp.2489-2497.

Ehbets, P.; Herzig H. P.; Prongue D. \& Gale M. T. (1992). High-efficiency continuous surfacerelief gratings for two-dimensional array generation. Opt. Lett., vol. 17, pp. 908-910.

Fienup, J. (1980). Iterative method applied to image reconstruction and to computergenerated holograms. Opt. Eng., vol. 19, pp. 297-306.

Fienup, J. (1982). Phase retrieval algorithms: a comparison. Appl. Opt., vol. 21, pp. 2758-2769.

Gale, M. T.; Lang G. K.; Raynor J. M.; Schutz H. \& Prongue D. (1992). Fabrication of kinoform structures for optical computing. Appl. Opt., vol. 31, pp. 5712-5715. 
Gale, M. T.; Rossi M.; Schutz H.; Ehbets P.; Herzig H. P. \& Prongue D. (1993). Continuousrelief diffractive optical elements for two-dimensional array generation. Appl. Opt., vol. 32, pp. 2526-2533.

Gallagher, N. C. \& Liu, B. (1973). Method for computing kinoforms that reduces image reconstruction error. Appl. Opt., vol. 12, pp. 2328-2335.

Gerchberg, R. W. \& Saxton, W. O. (1972). A practical algorithm for the determination of phase from image and diffraction plane pictures. Optik, vol. 35, pp. 237-246.

Herzig, H. P.; Prongue D. \& Dandliker R. (1990). Design and fabrication of highly efficient fan-out elements. Japanese J. of Appl. Phys., vol. 29, pp. L1307-L1309.

Johansson, M. \& Bengtsson, J. (2000). Robust design method for highly efficient beamshaping diffractive optical elements using an iterative-Fourier transform algorithm with soft operations. J. Mod. Opt., vol. 47, pp. 1385-1398.

Kuzmenko, A. V. (2006). Method of kinoform synthesis. UA Patent No. 65295 (priority from 03.07.2003), UkrPatent, Bulletin of Inventions No. 1, 2006.

Kuzmenko, A. V. \& Yezhov, P. V. (2007). Iterative algorithms for off-axis double-phase computer-generated holograms implemented with phase-only spatial light modulator. Appl. Opt., vol. 46, pp. 7392-7400.

Kuzmenko, A. V. (2008). Weighting iterative Fourier transform algorithm of the kinoform synthesis. Opt. Lett., vol. 33, pp. 1147-1149.

Kuzmenko, A. V. \& Yezhov, P. V (2008). Iterative Fourier-transform algorithm of synthesis of a kinoform with the use of the operation of predistortion of an object. Proc. of SPIE, vol. 7008, 70081W-1-9.

Kuzmenko, A. V. \& Yezhov, P. V (2009). Method of kinoform synthesis. UA Patent No. 86245 (priority from 05.02.2007), UkrPatent, Bulletin of Inventions No. 7, 2009.

Lee, W. H. (1979). Binary computer-generated holograms. Appl. Opt., vol. 18, pp. 3661-3668.

Levi, A. \& Stark, H. (1987). Restoration from phase and magnitude by generalized projections. Chapter 8 in Image Recovery: Theory and Application, ed. H. Stark, Academic Press, INC., 1987.

Lesem, L. B.; Hirsch P. M. \& Jordan J. A. Jr. (1967). Computer generation and reconstruction of holograms. Proc. Symp. Modern Optics, vol. 17 (New York: Polytechnic Institute of Brooklyn) p. 681-690.

Leger, J. R.; Chen D. \& Wang Z. (1994). Diffractive optical element for mode shaping of a Nd: YAG laser. Opt. Lett., vol. 19, pp.108-110.

Liu, J. S. \& Taghizaden, M. R. (2002). Iterative algorithm for the design of diffractive phase elements for laser beam shaping. Opt. Lett., vol. 27, pp. 1463-1465.

Lohmann, A. W. \& Paris, D. P. (1967). Binary Fraunhofer holograms generated by computer. Appl. Opt., vol. 6, pp. 1739-1748.

Mait, J. N. \& Brenner, K.-H. (1988). Optical symbolic substitution: system design using phase-only holograms. Appl. Opt., vol. 27, pp. 1692-1700.

Oton, J.; Ambs P.; Millan M. S. \& Perez-Cabre E. (2007). Multipoint phase calibration for improved compensation of inherent wavefront distortion in parallel aligned liquid crystal on silicon displays. Appl. Opt., vol. 46, pp. 5667-5679.

Prongue, D.; Herzig H. P.; Dandliker R. \& Gale M. T. (1992). Optimized kinoform structures for highly efficient fan-out elements. Appl. Opt., vol. 31, pp. 5706-5711.

Skeren, M.; Richter I. \& Fiala P. (2002). Iterative Fourier transform algorithm: comparison of various approaches. J. Mod. Opt., vol. 49, pp. 1851-1870. 
Tikhonov, A. N. \& Arsenin, V. IA. (1977). Solution of ill-posed problems, Halsted, New York.

Wyrowski, F. \& Bryngdahl, O. (1988). Iterative Fourier-transform algorithm applied to computer holography. JOSA A, vol. 5, pp. 1058-1065.

Wyrowski, F. (1990). Diffraction efficiency of analog and quantized digital amplitude holograms: analysis and manipulation. JOSA A, vol. 7, pp.383-393.

Wyrowski, F. (1990). Diffractive optical elements: iterative calculation of quantized, blazed phase structures. JOSA A, vol. 7, pp. 961-969.

Wyrowski, F. (1991). Upper bound of the diffraction efficiency of diffractive phase elements. Opt. Lett., vol. 16, pp. 1915-1917.

Wyrowski, F. \& Zuidema, R. (1994). Diffractive interconnection between a high-power ND:YAG laser and a fiber bundle. Appl. Opt., vol. 33, pp. 6732-6740.

Xin, T.; Gu B.-Y.; Yang G.-Z. \& Dong B.-Z. (1995). Diffractive phase elements for beam shaping: a new design method. Appl. Opt., vol. 34, pp.1314-1320.

Youla, D. C. \& Webb, H. (1982). Image restoration by the method of convex projections: Part 1 - Theory. IEEE Trans. On Medical Imaging, vol. MI-1, pp. 81-94. 


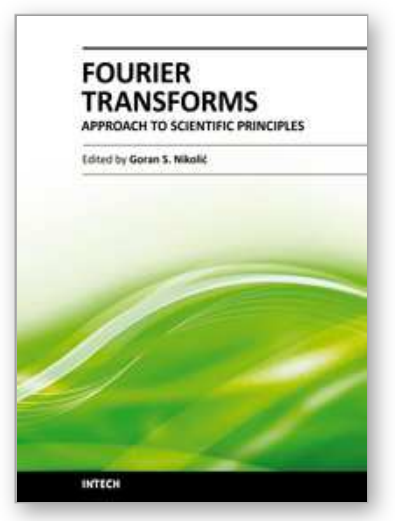

\author{
Fourier Transforms - Approach to Scientific Principles \\ Edited by Prof. Goran Nikolic
}

ISBN 978-953-307-231-9

Hard cover, 468 pages

Publisher InTech

Published online 11, April, 2011

Published in print edition April, 2011

This book aims to provide information about Fourier transform to those needing to use infrared spectroscopy, by explaining the fundamental aspects of the Fourier transform, and techniques for analyzing infrared data obtained for a wide number of materials. It summarizes the theory, instrumentation, methodology, techniques and application of FTIR spectroscopy, and improves the performance and quality of FTIR spectrophotometers.

\title{
How to reference
}

In order to correctly reference this scholarly work, feel free to copy and paste the following:

Alexander Kuzmenko, Pavlo lezhov and Jin-Tae Kim (2011). Weighting Iterative Fourier Transform Algorithm for Kinoform Implemented with Liquid-Crystal SLM, Fourier Transforms - Approach to Scientific Principles, Prof. Goran Nikolic (Ed.), ISBN: 978-953-307-231-9, InTech, Available from:

http://www.intechopen.com/books/fourier-transforms-approach-to-scientific-principles/weighting-iterativefourier-transform-algorithm-for-kinoform-implemented-with-liquid-crystal-sIm

\section{INTECH}

open science | open minds

\author{
InTech Europe \\ University Campus STeP Ri \\ Slavka Krautzeka 83/A \\ 51000 Rijeka, Croatia \\ Phone: +385 (51) 770447 \\ Fax: +385 (51) 686166 \\ www.intechopen.com
}

\author{
InTech China \\ Unit 405, Office Block, Hotel Equatorial Shanghai \\ No.65, Yan An Road (West), Shanghai, 200040, China \\ 中国上海市延安西路65号上海国际贵都大饭店办公楼 405 单元 \\ Phone: +86-21-62489820 \\ Fax: $+86-21-62489821$
}


(C) 2011 The Author(s). Licensee IntechOpen. This chapter is distributed under the terms of the Creative Commons Attribution-NonCommercialShareAlike-3.0 License, which permits use, distribution and reproduction for non-commercial purposes, provided the original is properly cited and derivative works building on this content are distributed under the same license. 